\title{
Nueva estructura para la enseñanza-aprendizaje de la materia de enfermería clínica en los estudios de Grado de Enfermería
}

\author{
Anna Falcó-Pegueroles, M. Teresa Luis-Rodrigo
}

Resumen. En el Grado de Enfermería, los cambios requeridos para la adaptación al Espacio Europeo de Educación Superior se han orientado a la introducción de novedades metodológicas que han propiciado la génesis de nuevos enfoques para la enseñanza y el aprendizaje, con el objetivo último de disminuir los desajustes que en algunas ocasiones se han detectado entre la teoría y la práctica. En esta línea, partiendo de las necesidades actuales de la formación en relación a la materia de enfermería clínica del grado y de las competencias profesionales, se inició un proceso de reflexión y análisis que originó una nueva estructura organizativa de la materia. Este nuevo enfoque nace con el objetivo fundamental de mejorar la docencia de la enfermería clínica, actualizando la forma de enseñar la enfermería y los cuidados enfermeros. Lejos del modelo biomédico centrado en la enfermedad que ha imperado hasta ahora, el nuevo enfoque parte de una visión profundamente enfermera y, en consecuencia, hace explícitos los cuidados de la persona con un trastorno real o potencial de la salud. Los resultados de la implementación de esta nueva estructura en la asignatura 'Enfermería clínica I' de segundo curso de grado ponen de manifiesto que hay una mejora en la precisión diagnóstica y en la etapa de planificación del proceso enfermero, así como en la identificación de la responsabilidad profesional en los ejes autónomo e interdisciplinar.

Palabras clave. Aprendizaje. Enfermería clínica. Enseñanza. Grado de Enfermería.

\section{A new structure for the teaching-learning of the subject of clinical nursing in Nursing Degree studies}

Summary. In Nursing Degree there have been many changes for adapting to the European Higher Education Area aimed to changing methodologies, but have also given rise to new approaches to teaching and learning. Their ultimate goal is to reduce the maladjustments have sometimes been found between theory and practice. Based on the education needs in the field of clinical nursing and also in professional competences, began a process of reflection and analysis resulted in a new organizational structure of matter. This new approach starts with the fundamental objective of improving the teaching of clinical nursing, updating the way we teach nursing and nursing care. Away from the biomedical model focused on the disease that has prevailed until now, the new approach comes from a nursing perspective, and therefore, makes explicit the care of the person with an actual or potential health disturbance. The results of implementing this new approach to the subject 'Clinical Nursing I' in second year of degree studies, show that the new structure represents an improvement in diagnostic accuracy and planning in the nursing process and the identification of responsibility in the independent and interdisciplinary professional axes.

Key words. Clinical nursing. Learning. Nursing Degree. Teaching.

\section{Introducción}

El nuevo paradigma universitario impulsado por las directrices del Espacio Europeo de Educación Superior (EEES) se centra principalmente en el aprendizaje del estudiante, hecho que ha generado la necesidad de vertebrar de forma explícita la formación universitaria actual a partir del modelo de educación basada en competencias [1-4].
Entre otros muchos cambios, esta línea de innovación ha generalizado la implementación de metodologías que convierten al estudiante en un sujeto activo y responsable de su propio aprendizaje. Estrategias como el aprendizaje basado en problemas, el portafolio de aprendizaje, la promoción de los seminarios, las tutorías, el aprendizaje cooperativo o la introducción de forma amplia de las tecnologías de la información y la comunicación a través de los cam-
Departamento de Enfermería Fundamental y Medicoquirúrgica. Escuela Universitaria de Enfermería y Podología. Campus de Ciencias de la Salud de Bellvitge. Universitat de Barcelona. L'Hospitalet de Llobregat, Barcelona, España.

Correspondencia: Anna Falcó Pegueroles. Departamento de Enfermería Fundamental y Medicoquirúrgica. Campus de Ciencias de la Salud de Bellvitge. Pavelló de Govern. Feixa Llarga, s/n. E-08907 L'Hospitalet de Llobregat (Barcelona).

E-mail:

annafalco@ub.edu

Financiación:

Proyecto de innovación docente reconocido en el marco del Programa de Mejora e Innovación Docente del Vicerrectorado de Política Docente y Científica de la Universitat de Barcelona (PID 2010 UB/48)

Conflicto de intereses: No declarado.

Conflict of interests: None declared.

(c) 2012 Educación Médica 
pus virtuales, con herramientas como la plataforma Moodle, están cada vez más presentes en los planes docentes de las universidades españolas y han demostrado un impacto positivo en el aprendizaje [5-9].

En el ámbito particular de la formación en enfermería, los cambios impulsados en el marco de la adaptación al EEES no sólo han sido en el plano metodológico, sino que también han introducido nuevos enfoques para la enseñanza y el aprendizaje [10]. Aunque, como apunta Canalejas [2], la formación enfermera siempre ha tomado en consideración las competencias profesionales a la hora de definir los contenidos y diseñar currículos que incluyeran una proporción importante de prácticas en el ámbito clínico, se observan aún en la actualidad algunos desajustes entre lo teórico y lo práctico. En esta línea, aunque en el contexto de la enseñanza profesional en Portugal, Soares et al [11] afirman que mientras la formación en el aula quiere enseñar al estudiante lo ideal, el ámbito asistencial le enseña lo posible en un entorno real, configurando un escenario complejo para el aprendizaje verdaderamente completo.

Ciertamente, se observa en nuestro ámbito algún desajuste cuando se relacionan determinados saberes teóricos con su poca aplicación a priori en el entorno asistencial. Un ejemplo concreto es la falta de consolidación en el ámbito clínico de la estructura y lenguaje enfermeros a la hora de diseñar los planes de cuidados y reflejarlos en la historia clínica. Ha sido en este contexto de cambio del paradigma universitario, con la consideración explícita y concreta de las competencias profesionales, donde se ha hecho más evidente este desajuste entre lo teórico y lo práctico en la formación en enfermería.

Se presenta en este artículo una nueva estructura para la enseñanza y el aprendizaje de la materia de enfermería clínica en los estudios de Grado de Enfermería que nace con el objetivo fundamental de mejorar la docencia de esta materia, actualizando la forma de enseñar enfermería y los cuidados enfermeros. Esta nueva estructura representa un cambio importante y se ha desarrollado mediante un complejo proceso reflexivo y analítico para reinventar la estructura organizativa y explicativa de la materia de enfermería clínica. La reflexión se centró fundamentalmente en tres aspectos:

- Competencias profesionales actuales, cuestión que permitió también explicitar las características diferenciales de la disciplina enfermera en el entorno sanitario actual.

- Paradigmas enfermeros, revisando los modelos teóricos vigentes y sus consecuencias para la práctica profesional.

- Método de razonamiento y lenguaje enfermeros.
El presente proyecto docente se inicia a partir de la experiencia, la creatividad y la vocación docentes de las profesoras coordinadoras de las asignaturas de 'Enfermería medicoquirúrgica I', de la Diplomatura de Enfermería, y de 'Enfermería clínica I', del Grado de Enfermería, y continúa con la profesionalidad de los docentes implicados, con voluntad constatada de trabajar en equipo para realizar un proyecto que repercute, de forma directa, en unos 380 alumnos de la asignatura de 'Enfermería clínica I' de segundo curso e, indirectamente, en otros 360 de tercer curso, de la Escuela Universitaria de Enfermería de la Universitat de Barcelona (UB).

Este proyecto de innovación docente se inició en la primavera del año 2009 y se consolidó durante el curso 2010-2011, momento en que comenzó en la Escuela de Enfermería de la UB la implementación de la asignatura en el segundo curso del Grado de Enfermería.

\section{Díficultad para enseñar cuidados enfermeros dentro de una estructura biomédica}

El objetivo fundamental de la materia de enfermería clínica del Grado de Enfermería, como lo era el de la materia de enfermería medicoquirúrgica de la diplomatura, es enseñar al estudiante a planificar los cuidados enfermeros que requiere la persona que padece o está en riesgo de padecer un trastorno de la salud. En consecuencia, son competencias específicas de la materia la valoración, la capacidad diagnóstica y la planificación de los cuidados de la persona adulta.

Esta materia curricular, que obviamente parte de una perspectiva holística del individuo, considera las repercusiones que tiene para la persona estar en la situación de pérdida de la salud, qué demandas o carencias se generan y qué cambios debe iniciar esta persona para adaptarse, mejorar su salud, tener una buena calidad de vida y ser lo más autónoma posible. Este es el centro y la razón de ser de la materia de enfermería clínica en la formación en enfermería.

En los estudios de diplomatura, la materia de enfermería medicoquirúrgica se desarrollaba en una estructura curricular que obligaba a situar en un primer plano la descripción de la enfermedad, la epidemiología, la sintomatología, las pruebas relacionadas y la terapéutica, para después explicar los cuidados enfermeros de la persona que la padecía. El estudiante se iniciaba en esta asignatura en el aprendizaje de la fisiopatología, los estudios diagnósticos, los tratamientos médicos y los cuidados en- 
fermeros, estos últimos como una parte más que se añadía al final y cuya extensión y profundidad dependía en muchas ocasiones del tiempo que el resto del programa permitiera. Los cuidados enfermeros, con la descripción de las complicaciones potenciales y los diagnósticos enfermeros más prevalentes relacionados, completaban una estructura que giraba en torno a la enfermedad y su descripción clínica.

Este modelo tradicional biomédico y la estructura que lo sustentaba hoy por hoy se consideran obsoletos porque dificultan la integración del pensamiento enfermero, además de evidenciar un desajuste entre el discurso teórico centrado en la persona y una estructura organizativa centrada en la enfermedad. Esto, sin contar con la incongruencia que subyace en el hecho de pretender enseñar cuidados enfermeros dentro de una estructura biomédica, perpetuando así una concepción de supeditación de la profesión enfermera a la profesión médica, en vez de ver la enfermería como lo que actualmente es: una profesión autónoma del ámbito de las ciencias de la salud.

Como ya apuntaba Pearson [12] en la década de los noventa, desde una perspectiva humanizadora de los cuidados, el modelo biomédico resulta incompleto y restrictivo para la enfermería, puesto que se centra en el diagnóstico médico, pone mayor énfasis en la tecnología y, dadas sus características, concentra la toma de decisiones 'en las manos de los médicos, por lo que para la enfermería es crucial el desarrollo de otras perspectivas' con las que poder prestar cuidados de forma completa y efectiva al servicio de la persona y la sociedad. De hecho, los modelos enfermeros no centran su atención en la enfermedad o alteración de la salud que sufre la persona, sino que analiza sus repercusiones físicas, psíquicas, sociales y espirituales, lo que sirve de base a la enfermería para diseñar y poner en práctica estrategias concretas con el objetivo de ayudar al paciente y a su familia a conseguir el máximo grado de salud y bienestar posibles.

Por este motivo, el profesional enfermero tiene que capacitarse para prestar cuidados integrales a la persona y debe utilizar metodología propia [13] para describir los cuidados vinculados a la persona y no a la enfermedad, lo que hace necesario que el proceso de enseñanza y aprendizaje tenga una estructura que refleje el razonamiento profesional enfermero. Afortunadamente, la entrada al EEES ha dado a los docentes la posibilidad de cuestionar y cambiar estos desajustes para mejorar la enseñanza y el aprendizaje en la formación enfermera.

Así pues, a partir de esta evidencia, en la Escuela de Enfermería de la UB iniciamos un proceso de re- flexión que llevó a centrarnos en las competencias del profesional de enfermería, en concretar cómo razonaba, qué áreas de responsabilidad tenía y cómo se organizaba la enfermera clínica en el ámbito asistencial. Como afirman varios autores $[1,3]$, identificar las necesidades formativas y establecer los resultados del aprendizaje es el primer paso para planificar un programa formativo.

Éste fue el punto de partida de un proceso reflexivo sobre la aportación diferencial de la enfermería al equipo sanitario y sobre las competencias que debían asumir los estudiantes de enfermería en relación a la materia de enfermería clínica del grado.

\section{Nueva estructura para la materia de enfermería clínica}

La nueva estructura para la enseñanza y el aprendizaje distribuye los contenidos de las unidades temáticas en tres temas básicos. Debe tenerse en cuenta que el objetivo final es explicar los cuidados enfermeros de la persona con un trastorno real o potencial de la salud, explicitando el pensamiento y organización enfermera. Este proceso conlleva partir de una estructura transversal que agrupe aquellos aspectos de las diversas patologías que, desde la perspectiva enfermera, requieren cuidados semejantes y por tanto pueden asociarse, a pesar de constituir entidades fisiopatológicas distintas.

Los contenidos de la unidad temática se distribuyen, pues, en los temas siguientes (Tabla I):

\section{Tema 1. Trastornos del aparato $X$}

Resumen de la fisiopatología de los trastornos más prevalentes en nuestro entorno clínico del aparato o sistema corporal de que se trate, destacando de manera especial la valoración de la persona afecta$\mathrm{da}$, las repercusiones que la sintomatología tiene en las actividades de la vida diaria y las implicaciones para la enfermera responsable de su cuidado.

Tratamiento médico habitual, con especial énfasis en las consideraciones prácticas que debe tener en cuenta el profesional enfermero y no en la justificación de la prescripción de la terapéutica médica o en las características farmacocinéticas. Cabe recordar que el estudio de las pruebas diagnósticas se incluye en la asignatura de 'Fisiopatología'.

\section{Tema 2. Rol de colaboración}

Este apartado se refiere al ámbito interdisciplinar o de colaboración con otros profesionales, general- 
Tabla I. Diferencias entre la estructura de la enfermería medicoquirúrgica (Diplomatura de Enfermería) y la nueva estructura propuesta para la materia de enfermería clínica en el Grado de Enfermería.

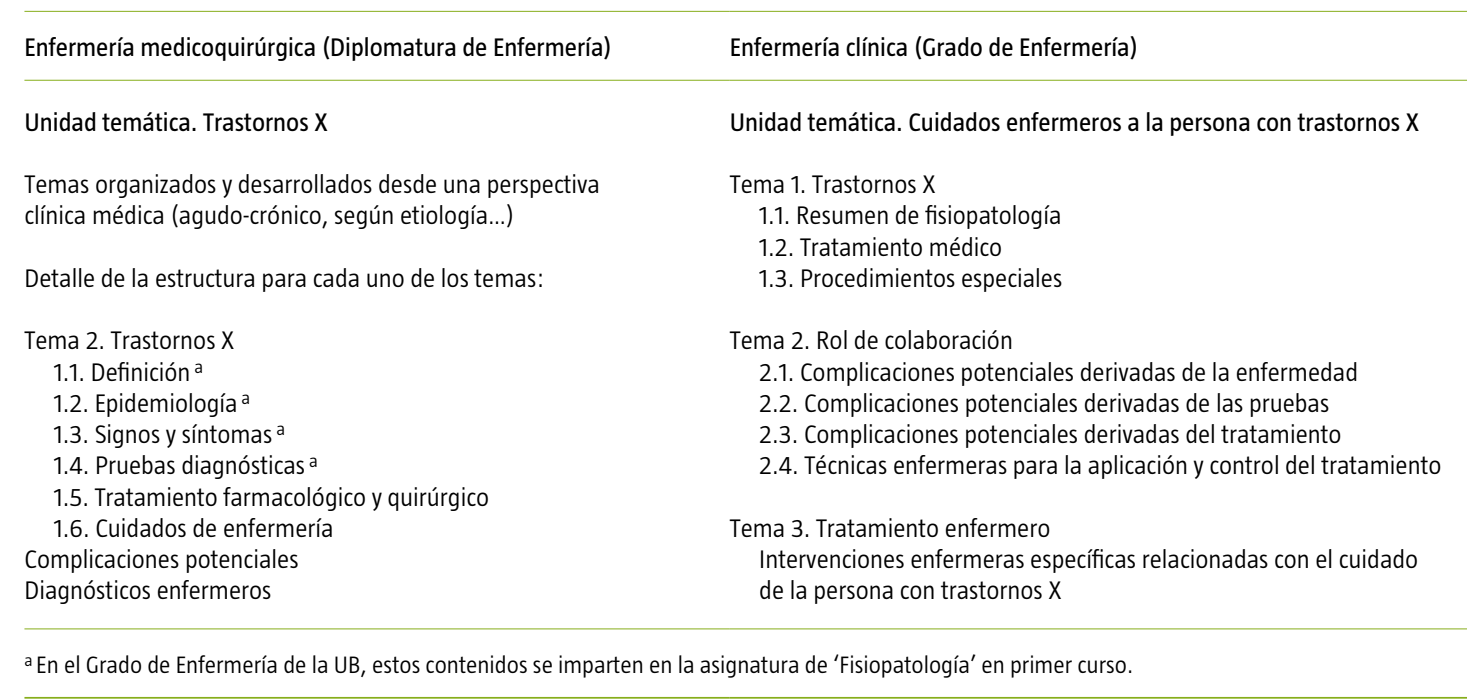

mente el médico. En primer lugar, se identifican las complicaciones potenciales más prevalentes relacionadas con la enfermedad, las pruebas o procedimientos diagnósticos y el tratamiento médico [14]. En segundo lugar, se incluyen los procedimientos enfermeros necesarios para controlar la situación de salud, prevenir las complicaciones y aplicar el tratamiento médico prescrito.

El objetivo de este tema es que el estudiante aprenda a identificar y formular correctamente las complicaciones potenciales más habituales, y a desarrollar de forma completa las actividades enfermeras que debería llevar a cabo para prevenirlas y detectarlas precozmente.

\section{Tema 3. Tratamiento enfermero}

Este tema se centra en las intervenciones enfermeras específicas relacionadas con la persona que tiene o está en riesgo de padecer un trastorno de la salud abordado en el tema 1. El objetivo es que el estudiante aprenda a identificar la que debería ser su aportación específica al cuidado de la salud de las personas, señalando en cada situación tanto las intervenciones más adecuadas como las actividades más habituales para cada intervención.

Es en el contexto de los seminarios, con grupos reducidos y mediante ejercicios y trabajos cooperativos, donde se analizan casos clínicos que abordan situaciones individuales y permiten centrar la atención del estudiante en la respuesta de la persona frente a los procesos vitales o problemas de salud reales o potenciales, lo que constituye el centro de atención de los diagnósticos enfermeros [14]. El objetivo de los seminarios es potenciar en el estudiante el razonamiento clínico enfermero y desarrollar su capacidad y precisión diagnósticas.

Como muestra la tabla I, los contenidos relacionados con los cuidados enfermeros, tanto en el eje autónomo como en el interdependiente, se presentan de forma explícita y constituyen la estructura básica de la enfermería clínica. Su proporción es mayor en el grado de lo que lo era en la diplomatura, puesto que la nueva estructura curricular introduce al estudiante en la materia de fisiopatología ya en primer curso, permitiendo que en el segundo curso el aprendizaje se centre en los cuidados enfermeros. En consecuencia, la distribución temporal de los contenidos es la siguiente:

- Tema 1: 30-35\% de las horas totales.

- Temas 2 y 3: 65-70\% de las horas totales.

\section{Metodología y sistema de evaluación de la asignatura}

La materia de enfermería clínica del Grado de Enfermería de la UB tiene un total de 18 ECTS (European Credit Transfer System), divididos entre segundo y tercer curso de grado. En lo que respecta específicamente a 'Enfermería clínica I' (segundo 
Tabla II. Diferencias y semejanzas entre las asignaturas de 'Enfermería medicoquirúrgica I’ y 'Enfermería clínica I’.

\begin{tabular}{|c|c|c|}
\hline & Enfermería medicoquirúrgica I & Enfermería clínica I \\
\hline Titulación universitaria (duración) & Diplomatura de Enfermería (3 años) & Grado de Enfermería (4 años) \\
\hline Estado actual & En proceso de extinción & En proceso de implementación \\
\hline Curso académico & Segundo & Segundo \\
\hline \multirow{2}{*}{ Créditos y su distribución } & 9 créditos universitarios & 9 créditos ECTS \\
\hline & 6 teoría - 3 prácticos & 3,6 presencial $-2,4$ trab. dirigido -3 trab. autónomo \\
\hline Especificidad & - & Unida a 3 créditos de 'Dietética humana' \\
\hline Categoría & Troncal semestral & Troncal anual \\
\hline $\begin{array}{l}\text { Asignaturas previas } \\
\text { relacionadas (1er. curso) }\end{array}$ & $\begin{array}{l}\text { Estructura y función del cuerpo humano } \\
\text { Introducción a la enfermería medicoquirúrgica }\end{array}$ & Fisiopatología humana \\
\hline $\begin{array}{l}\text { Asignatura posterior } \\
\text { relacionada (3 }{ }^{\text {rer }} \text { curso) }\end{array}$ & Enfermería medicoquirúrgica II & Enfermería clínica II \\
\hline \multirow[b]{2}{*}{ Objetivos generales } & $\begin{array}{l}\text { Identificar los trastornos fisiopatológicos } \\
\text { asociados a órganos y sistema }\end{array}$ & \multirow{2}{*}{$\begin{array}{l}\text { Diseñar planes de cuidados adaptados a la } \\
\text { persona que padece o está en riesgo de padecer } \\
\text { un trastorno de la salud }\end{array}$} \\
\hline & $\begin{array}{l}\text { Diseñar planes de cuidados adaptados } \\
\text { a la persona que padece o está en riesgo } \\
\text { de padecer un trastorno de la salud }\end{array}$ & \\
\hline Perfil de los docentes & $\begin{array}{l}\text { Expertos en enfermería medicoquirúrgica } \\
\text { y en docencia universitaria }\end{array}$ & $\begin{array}{l}\text { Expertos en enfermería clínica } \\
\text { y en docencia universitaria }\end{array}$ \\
\hline
\end{tabular}

curso de grado), y de acuerdo con el plan de estudios vigente, a los nueve créditos anuales de la materia se le añaden tres créditos de 'Dietética humana', sumando la asignatura un total de 12 créditos. De los nueve créditos específicos (que representan un total de 225 horas), 90 horas son de actividad presencial en el aula, 60 horas son de trabajo dirigido y 75 horas, de aprendizaje autónomo. Pueden observarse en la tabla II las principales diferencias y semejanzas entre la nueva asignatura del Grado de Enfermería y la asignatura anterior impartida en la Diplomatura de Enfermería.

Respecto a la metodología, el proyecto docente se implementa, fundamentalmente, mediante clases expositivas y seminarios en grupos reducidos, pero también a través de ejercicios y cuestionarios en el campus virtual, además del seguimiento tutorial periódico. También se facilita a los estudiantes el material de las sesiones, los objetivos de aprendizaje para guiar el trabajo autónomo, así como bibliografía con artículos y direcciones web de sociedades científicas o instituciones sanitarias.
La evaluación acoge los sistemas de evaluación continuada o de evaluación única. El estudiante, una vez informado de la dinámica de cada uno de estos sistemas, debe escoger una u otra opción. En relación a la evaluación continuada, el $60 \%$ de la nota se obtiene a partir de tres pruebas escritas individuales tipo test, y el $40 \%$ restante, a través de los trabajos específicos vinculados a los seminarios grupales e individuales, un total de diez a lo largo del curso. Respecto a la evaluación única, se realiza a partir de una prueba escrita final que incluye preguntas tipo test, preguntas a desarrollar, y el análisis y resolución de un caso. Mayoritariamente, los estudiantes se acogen a la evaluación continuada y sólo un 2-5\% a la evaluación única.

\section{Resultados preliminares}

La implementación de la nueva estructura durante el curso 2010-2011 ha puesto de manifiesto una mejora en el aprendizaje del estudiante en lo que se 
refiere a su capacidad de precisión en las fases de valoración, diagnóstico y planificación del proceso enfermero, así como en la identificación de la responsabilidad profesional en los ejes autónomo e interdisciplinar. Esta mejora se ha evidenciado a través del análisis de los resultados de los ejercicios, los casos clínicos y las pruebas escritas que se han realizado durante el curso.

En la actualidad se está estudiando el impacto de la nueva estrategia, aunque los resultados de la evaluación final de los estudiantes ya indican un porcentaje de aprobados del $87 \%(n=307)$, de los cuales el 33\% alcanza una calificación de notable. Es un porcentaje claramente superior al de la asignatura de enfermería medicoquirúrgica, con un $65-70 \%$ de aprobados en primera convocatoria, en función de los grupos de docencia. Aun así, a la espera de un análisis multivariante más exhaustivo, esta mejora en los resultados se asocia, en primer lugar, al método de evaluación continuada seguido durante el curso.

En el aspecto cualitativo, los docentes implicados en el proyecto hemos constatado que la nueva estructura se adapta perfectamente al objetivo fundamental de la asignatura y que es una estructura coherente y basada en un enfoque profundamente enfermero, lo que nos ha permitido centrarnos en los contenidos verdaderamente relevantes para enseñar al estudiante a diseñar planes de cuidados.

\section{Conclusiones}

Uno de los desajustes identificados por los agentes implicados en la formación universitaria en enfermería es la discrepancia entre la enseñanza teórica que tiene lugar en las aulas y lo que la enfermera puede realmente llevar a cabo en un entorno clínico con los recursos y las características de cada institución sanitaria. No decimos nada nuevo si afirmamos que resulta complejo enseñar al estudiante cómo razona la enfermera en el entorno asistencial, y es a su vez extremadamente difícil hacerlo sin utilizar de forma explícita la estructura de pensamiento y lenguaje enfermeros.

El nuevo modelo que proponemos para la enseñanza y el aprendizaje de los cuidados enfermeros, dirigido tanto a las personas sanas como a las que padecen algún tipo de trastorno o alteración fisiopatológica, se basa en el razonamiento clínico o razonamiento enfermero, lo que permite minimizar los desajustes entre aspectos teóricos y prácticos de la enfermería clínica. Las razones son básicamente dos: en primer lugar, el nuevo modelo se centra en la persona y no en la situación fisiopatológica que padece o está en riesgo de padecer; característica que lo distancia claramente del modelo biomédico, hoy por hoy obsoleto. En esta línea, ha sido necesaria una reestructuración de los contenidos orientada por las competencias profesionales. En otras palabras, esta reestructuración permite hacer más explícita el área de competencia enfermera y detallar aspectos fundamentales del plan de cuidados, aproximándose más a la realidad profesional actual y a las demandas de la sociedad.

En segundo lugar, y en aras a fomentar la coherencia, se utiliza el lenguaje enfermero de forma central y explícita, introduciendo el estudiante en la terminología enfermera profesional desde el principio y a partir de la experimentación y no desde la descripción.

En definitiva, estamos convencidos de que este nuevo modelo mejorará la adquisición de las competencias específicas en relación a la materia de enfermería clínica definidas en el Grado de Enfermería.

\section{Bibliografía}

1. Falcó A. La nueva formación de profesionales: sobre la competencia profesional y la competencia del estudiante de enfermería. Educ Med 2004; 7: 42-5.

2. Canalejas MC. Enseñanza de Grado en Enfermería en el marco de la convergencia europea. Metas de Enfermería 2006; 9: 28-31.

3. Palés JL. Planificar un currículum o un programa formativo. Educ Med 2006; 9: 59-65.

4. Palés J, Nolla M, Oriol A, Gual A. Proceso de Bolonia (I) educación basada en competencias. Educ Med 2010; 13: 127-35.

5. Betolaza E, Alonso I. El diario reflexivo y el autoaprendizaje tutorizado. Metas de Enfermería 2002; 45: 14-8.

6. Vila M, Vilanova M, Mas N. Seminarios con casos simulados en la diplomatura de enfermería. Educ Med 2002; 5: 184-90.

7. Baños JE, Pérez J. Cómo fomentar las competencias transversales en los estudios de ciencias de la salud: una propuesta de actividades. Educ Med 2005; 8: 216-25.

8. Serrano P, Martínez M, Arroyo MP, Lanza D. Análisis del portafolio como herramienta evaluativa de las prácticas clínicas de enfermería comunitaria en estudiantes de grado. Educ Med 2010; 13: 177-85.

9. Canalejas MC. El portafolio como herramienta didáctica: un estudio en escuelas universitarias de enfermería. Educ Med 2010; 13: 53-61.

10. Falcó A. Orientar la asignatura de anatomía y fisiología hacia las competencias profesionales de enfermería. Propuesta de un proyecto docente. Educ Med 2005; 8: 208-15.

11. Soares SM, Silva MA, Schubert VM, Fontoura MH. Formación clínica en enfermería comunitaria. Revista Rol de Enfermería 2010; 33: 504-10.

12. Pearson A, Vaughan B, Fitzgerald M. Nursing models for practice. 2 ed. Edinburgh: Butterworth-Heinemann; 2004.

13. Martínez ML. Treinta años de evolución de la formación enfermera en España. Educ Med 2007; 10: 93-6.

14. Luis MT. Los diagnósticos enfermeros. Revisión crítica y guía práctica. 8 ed. Barcelona: Elsevier-Masson; 2008. 